\title{
Evaluación de los procesos financieros con base en el modelo PEF en una institución de educación superior ${ }^{*}$
}

\author{
Assessment of financial processes based on the PAF \\ model in a Higher Education Institution
}

\section{Avaliação dos processos da área financeira baseado no modelo PEF em uma Instituição de Ensino Superior}

Recibido: 21 de diciembre de 2018

Revisado: 12 de febrero de 2019

Aceptado: 8 de marzo de 2019

\begin{abstract}
Rossy Esperanza Quintana Machado**
Universidad Santo Tomás, Colombia

Cómo citar: Quintana Machado, R.E. (2019). Evaluación de los procesos del área financiera con base en el modelo PEF en una institución de educación superior. Signos. Investigación en Sistemas de Gestión, 11(2), 171-180. Doi: https://doi.org/10.15332/24631140.5088
\end{abstract}

\section{RESUMEN}

El presente artículo describe los principales aspectos que se tuvieron en cuenta para evaluar los procesos del área financiera de una institución de educación superior y su articulación con el sistema de gestión de la calidad con base en el modelo PEF (prevención, evaluación y fallas). Inicialmente se realizaron reuniones con académicos, como usuarios directos de los procesos, donde se escucharon de manera objetiva los puntos de vista a través de lluvia de ideas. Se culminó con una sugerencia desde la perspectiva de dichos usuarios. Se usó una metodología mixta, que involucró la parte cuantitativa, al valorar las fallas en los procesos, y la cualitativa, al medir el nivel de insatisfacción de los usuarios internos por la demora o el incumplimiento en

\footnotetext{
* Artículo de investigación.

** Magíster en Calidad y Gestión Integral, especialista en Auditoría de Sistemas, especialista en Gerencia de Instituciones de Educación Superior, contadora pública. Bogotá, Colombia. Correo electrónico: rossyquintana@usantotomas.edu.co. Orcid: https://orcid.org/0000-0002-6234-3215
} 
los diversos procesos que maneja el área. Los principales resultados muestran, después de realizar el análisis de cada uno de los procesos establecidos (planeación matrícula, área de crédito, pagaduría, tesorería y movilidades), una calificación muy baja (crítica) en dos procesos considerados los más sensibles y de urgente intervención: el caso de honorarios/OPS y el de movilidad. Por lo anterior se propone un plan de mejora que permite identificar los beneficios económicos y financieros del sistema de gestión de la calidad, que garantiza la optimización en los procesos.

Palabras clave: evaluación, modelo PEF, sistema de gestión de la calidad, costes de la calidad.

\section{ABSTRACT}

This paper describes the main aspects that were taken into account to assess the financial area processes of a Higher Education Institution and their articulation with the quality management system based on the Prevention, Appraisal and Failure (PAF) model. Initially, meetings were held with the Academy, as direct users of the processes, at which the different points of view were objectively listened to, in a brain storming session, concluding with a suggestion from their view as academicians and main users. The approach was based on a mixed methodology that involved the quantitative part, by estimating failure costs in processes, and qualitative, measuring the dissatisfaction degree of internal users due to the delay or noncompliance with the different processes managed in the area. The main results show, after analyzing each one of the processes established (registration planning, credit area, payment, treasury and mobility), a very low (critical) score in two processes considered the most sensitive ones and requiring urgent intervention, such as the fees/OPS and the mobility processes; the forgoing entails an improvement plan intended to identify the economic and financial benefits of the quality management system and that guarantees process optimization.

Keywords: Assessment, PAF model, Quality Management System, quality costs

\section{RESUMO}

Este artigo descreve os principais elementos levados em conta na avaliação dos processos da área financeira de uma Instituição de Ensino Superior e sua vinculação com o sistema de gestão da qualidade baseado no modelo de Prevenção, Avaliação e Falhas (PEF, por suas siglas em espanhol). Primeiramente, realizaramse reuniões com a academia, como os usuários diretos dos processos, onde se escutaram objetivamente os pontos de vista, através de brainstorming, encerrando com uma sugestão da sua visão de acadêmicos e usuários principais. Empregou-se uma metodologia mista, envolvendo a parte quantitativa, custeando as falhas nos processos e a parte qualitativa, medindo o nível de insatisfação de usuários internos pelo retardo ou inadimplência nos diversos processos que trata dessa área. Os principais resultados revelam, depois de executar a análise de cada um dos processos estabelecidos (planejamento, inscrição, área de crédito, pagamentos, tesouraria e mobilidades), uma qualificação muito baixa (crítica) em dois processos considerados os mais sensíveis e urgentes de intervenção, nomeadamente, o caso do processo de estipêndios/OPS e de mobilidade. $O$ anterior propõe um plano de melhoria que possibilite a identificação dos benefícios econômicos e financeiros do sistema de gestão de qualidade e que garanta 0 aprimoramento dos processos.

Palavras-chave: Avaliação, Modelo PEF, Sistema de gestão da qualidade, custos da qualidade. 


\section{INTRODUCCIÓN}

La calidad cada vez cobra mayor valor en la gestión educativa, así que se requieren métodos y recursos para optimizar la gestión (Águila, 2005) dado el incremento en la oferta de IES (Instituciones de Educación Superior) y la preocupación de estas por su productividad (RuelasBarajas, 1993). En este sentido, y con el ánimo de argumentar la importancia de los sistemas de gestión de la calidad, se requiere el análisis de los costos de calidad y de la falta de esta (Perdomo, 2010).

En Colombia a partir de la Ley 30 de, 1992 se propende a la creación de mecanismos de evaluación de la calidad de los programas académicos, y en el Decreto 1295 de 2010 se precisan los requisitos asociados a las condiciones de calidad compilados en el Decreto 1075 de 2015. Lo anterior evidencia la importancia del tema de la calidad en las IES en este país. Por consiguiente, algunas investigaciones (Olmos, 2012; Pedraza, Rodríguez y Pérez, 2017) han buscado identificar aquellos requisitos articuladores entre los sistemas de gestión de la calidad y la gestión educativa, asícomo las variables centrales para evaluar la gestión. Esto con el fin de encontrar elementos que faciliten el cumplimiento de la legislación colombiana y la apropiación de este sistema de gestión por parte de las IES en pro de la mejora continua de sus procesos y del desempeño del referido sistema.

Cabe precisar que no solo se requiere alinear los requisitos de estos modelos de gestión de la calidad, sino que además es imperativo comprender los costos asociados a la calidad y la falta de esta. El monitoreo de los indicadores de calidad económica es un mecanismo que facilita el control empresarial y a la vez, una herramienta de soporte de gestión de calidad dado que permite la optimización de costos, procesos y productos de mejora de la calidad. Ello redunda, por lo tanto, en la satisfacción del cliente (Satanova y Sedliacikova, 2015).
De acuerdo con la revisión de literatura, el modelo PEF se desarrolló para estimar los costos de calidad (Gracia y Dzul, 2007; Lozano, Keith y Fonseca, 2014). Este modelo considera costos de prevención, de evaluación y de fallas (Perdomo, 2010). Los costos de prevención son las actividades realizadas para evitar defectos en el diseño y desarrollo en compras, equipos, instalaciones y materiales, y en todo el proceso de generación del producto o servicio. Los costos de evaluación corresponden a los procesos de auditoría para determinar la conformidad del producto o servicio, las inspecciones y las pruebas de materiales, entre otros. Y los costos asociados a las fallas, que pueden ser internas o externas, atienden a los desperdicios y reprocesos generados, a las quejas recibidas por parte de los clientes y a procesos de devolución, entre otros (Gracia y Dzul, 2007).

En este sentido, el presente artículo muestra los resultados de una investigación con la que se buscó evaluar los procesos financieros de una IES de la ciudad de Bogotá, su cuantificación a través del método PEF y a la vez su articulación con el sistema de gestión de la calidad. A partir de allíse tomarán decisiones de peso para establecer las posibles mejoras en los procesos que se determinen críticos y que necesiten una intervención.

En el plano teórico este estudio de caso servirá para realizar un acercamiento a la alta gerencia de la IES en cuestión. Posteriormente esta será informada de manera clara y sucinta acerca de la importancia de mejorar los procesos del Área Financiera y conocer la percepción de los académicos (decanos de División, decanos de Facultad) como usuarios directos de estos procesos, teniendo en cuenta que la universidad se encuentra actualmente certificada con la ISO 9001.

De otro lado, es importante resaltar que la IES estudiada involucra en su gestión los principios de la responsabilidad social, dentro de los cuales se encuentran: 
transparencia, comportamiento ético, legalidad y respeto a los intereses de las partes involucradas, a la normativa internacional de comportamiento y a los derechos humanos. Todo lo anterior se hace necesario para la adopción de un sistema gerencial o de mejoras con orientación a la calidad, que favorezca los logros y objetivos establecidos y haga más competitiva la gestión de la universidad.

El presente proyecto investigativo tiene una metodología mixta, que involucra la parte cuantitativa, al valorar las fallas en los procesos, y cualitativa, al medir el nivel de insatisfacción de los clientes internos por la demora o incumplimiento en los diversos procesos que maneja el Área de Gestión Financiera de la universidad.

De acuerdo con lo anterior, el presente estudio tiene como objetivo proponer un plan de mejora que permita identificar los beneficios económicos y financieros del sistema de gestión de la calidad y garantice la optimización de los procesos del Área Financiera de la IES objeto de estudio. Asimismo, se seleccionan los procesos del Área Financiera de la universidad para realizar el análisis del costo de la calidad y la falta de esta a través del modelo PEF, calcular con exactitud los costos de la calidad y la falta de esta en los procesos críticos y articular los procesos del Área de Gestión Financiera con el sistema de gestión de calidad de la IES.

Este diseño busca entender las diferentes percepciones de los académicos con base en sus experiencias como usuarios funcionales de los procesos del Área de Gestión Financiera. Para lograr esto se realizaron reuniones con aportes objetivos de tales usuarios basados en datos reales, se analizaron los resultados sistemáticamente y, a través de una calificación ponderada, se evaluó cada proceso para determinar el costo de las fallas de manera objetiva.

\section{METODOLOGÍA}

La investigación se desarrolla buscando establecer relaciones causa-efecto con el fin de evaluar los procesos del Área Financiera de la IES en cuestión y su articulación con el sistema de gestión de calidad con base en el modelo PEF. Eso debe servir para detectar fallas en los procesos y establecer cuánto valen. Tales fallas afectan directamente la rentabilidad o el crecimiento de las utilidades, así que es normal que la alta gerencia se preocupe por conocer cómo puede mejorar sus procesos y asílograr una mayor productividad y satisfacción de sus clientes tanto internos como externos.

Cuantificar el resultado de identificar los procesos más críticos del Área Financiera (valoración cuantitativa) nos sirve como base para aplicar el método PEF. Asimismo, al medir las fallas en nuestros procesos se evidencia un costo no recuperable, el costo reputacional. Por lo tanto, estaremos frente a una evaluación cualitativa. Por esta razón la presente investigación es mixta.

Se reconoce que la investigadora tiene conocimiento de las debilidades en los procesos del Área de Gestión Financiera de la IES por estar vinculada laboralmente a dicha área. Sin embargo, esta investigación no pretende el levantamiento de procesos plenamente identificados, sino la construcción de conocimiento a partir de la racionalidad y la lógica de la generación de unos lineamientos que sirvan para articular tales procesos con el sistema de gestión de calidad de la IES bajo estudio.

Con base en lo anteriormente expuesto, la información para llevar a cabo esta investigación se recolectó a partir de reuniones de trabajo desarrolladas bajo los siguientes lineamientos:

1) Se solicitó agenda con cada uno de los académicos, que se encuentran agrupados por áreas del 
conocimiento, incluidos los decanos de división y sus decanos de facultad, quienes tienen poder decisorio sobre los procesos a nivel de recomendaciones de mejora; la asistente de investigación, quien colaboró registrando las calificaciones en la matriz de trabajo de campo, en forma simultánea para evitar el sesgo de error y acorde con una escala de valores previamente establecida, y obviamente la investigadora, quien coordinaba todas las reuniones de trabajo. Cabe precisar que algunas reuniones fueron grupales y otras, individuales.

2) En cuanto a la población del estudio se decidió trabajar con una muestra de seis divisiones: Ciencias Económicas y Administrativas, Ingenierías, Ciencias Sociales, Ciencias de la Salud, Ciencias Jurídicas y Políticas, y Teología y Filosofía.

3) La información se recolectó a partir de 16 reuniones de trabajo, de las cuales 13 fueron individuales y 3 grupales, con 26 personas involucradas entre decanos de división y de facultad. La primera reunión que se realizó en forma grupal fue con la División de Ciencias Económicas y Administrativas, donde participaron los decanos de las facultades de Contaduría, Negocios Internacionales, Administración de Empresas y Estadística. La reuniones con la División de Ingenierías se realizaron en forma individual y participaron los decanos de las facultades de Ingeniería Civil, Ingeniería Ambiental y Telecomunicaciones. Igualmente, con las divisiones de Ciencias Sociales y de la Salud se realizaron reuniones en forma individual y participaron los decanos de las facultades de Comunicación Social, Diseño Gráfico y Sociología, en el primer caso; y de Sicología, Cultura Física, Recreación y Deporte, en el segundo. Se finalizó realizando dos reuniones grupales, una con la División de Ciencias Jurídicas y Políticas y otra con la División de Filosofía y Teología.

4) Lo siguiente que se hizo fue crear la matriz de trabajo de campo, donde se recopilaron los comentarios asociados a cada proceso. Algunos propusieron acciones de mejora, las cuales se tuvieron en cuenta para las recomendaciones finales. Acto seguido se asignó la puntuación o calificación para cada uno de los criterios establecidos en la matriz y después de obtener la calificación en cada proceso se realizó la media o promedio para de esta forma establecer cuáles de los procesos son considerados críticos 0 que requieren intervención.

El objetivo de las reuniones fue conocer la percepción tanto de los decanos de división (padres) como de los decanos de facultad (civiles) acerca de los procesos del Área Financiera de la Universidad. Para llegar a esto se realizó el levantamiento de dichos procesos, que se presentó a todos y cada uno de los participantes en las reuniones de trabajo con el fin de obtener una calificación, la cual fue registrada por la asistente de la investigación en la correspondiente matriz de análisis de datos. Esta actividad se realizó en forma paralela dentro de las reuniones así: los decanos, según su percepción y la escala establecida, realizaban la correspondiente ponderación. En el caso de las reuniones grupales tal ponderación implicaba el consenso de los participantes con el fin de evitar sesgos en los registros. Es decir, a los comentarios de los decanos se les dio un tratamiento, mediante una escala de valoración y como producto de las reuniones de trabajo, centrado en la ponderación de los procesos, lo cual permitió graficar y calcular los promedios que evidencian cuáles de los procesos tienen calificación baja, según la opinión de los académicos. 


\section{RESULTADOS Y DISCUSIÓN}

En la tabla 1 se establece una escala de valoración para cada uno de los procesos así: los que estuvieron entre 0 y 25 puntos pertenecen al nivel bajo; los que obtuvieron entre 26 y 50, al nivel medio bajo; los que estuvieron entre 51 y 75, al nivel medio alto y los que obtuvieron por encima de 75, al nivel alto.

Tabla 1. Escala de valoración

\begin{tabular}{|l|l|}
\hline \multicolumn{1}{|c|}{ Niveles } & \multicolumn{1}{c|}{ Puntuación } \\
\hline Bajo & Entre 0 y 25 \\
\hline Medio bajo & Entre 26 y 50 \\
\hline Medio alto & Entre 51 y 75 \\
\hline Alto & Entre 76 y 100 \\
\hline
\end{tabular}

Fuente: elaboración propia.

\section{Procesos del Área de Gestión Financiera}

Se realizó el levantamiento de cada uno de los procesos macro o principales que conforman el Área de Gestión Financiera de la universidad, con sus respectivos subprocesos y procedimientos.

Al calcular el coste de uno de los procesos críticos, el de pago de honorarios, los resultados son los siguientes: intervienen once dependencias de la Usta y durante un año los costos de calidad ascienden a $\$ 453842992$ y los de falta de calidad, a $\$ 77333055$, más el daño reputacional o de buen nombre de la universidad, que es intangible.

Con base en el modelo PEF se determinó qué hacer en cuanto a prevención, detección y control. Después del cálculo de costo por hora se procede a relacionar las causas de las fallas, evidenciándose las siguientes:

- Error en el diligenciamiento de las planillas de movilidades: $10 \%$.

- Cambio de agenda para los ponentes: $10 \%$.

- Solicitudes mal diligenciadas (fechas, horas): $40 \%$.

- No contactar al viajero: $30 \%$.

- Demora en la confirmación: $45 \%$.

- Cambio agenda de ponencias: $10 \%$.

\section{Análisis de los procesos por división}

En la tabla 2 se explica cómo al aplicar la escala de valores se estableció el promedio de calificación asignado a cada proceso según los criterios y observaciones emitidas por las decanaturas de cada división.

Tabla 2. Calificación de cada proceso

\begin{tabular}{|l|c|c|c|c|c|c|c|}
\hline \multicolumn{1}{|c|}{ Proceso macro } & $\begin{array}{c}\text { Administrativas } \\
\text { y contables }\end{array}$ & $\begin{array}{c}\text { División de } \\
\text { Ingenierías }\end{array}$ & $\begin{array}{c}\text { División de } \\
\text { Ciencias } \\
\text { Sociales }\end{array}$ & $\begin{array}{c}\text { División de } \\
\text { Ciencias } \\
\text { de la Salud }\end{array}$ & $\begin{array}{c}\text { División de } \\
\text { Ciencias Jurídicas } \\
\text { y Políticas }\end{array}$ & $\begin{array}{c}\text { División de } \\
\text { Filosofía y } \\
\text { Teología }\end{array}$ & Promedio \\
\hline Planeación matrícula & 76 & 69 & 88 & 80 & 55 & 60 & 73 \\
\hline Área de Crédito & 96 & 67 & 72 & 75 & 65 & 65 & 69 \\
\hline Pagaduría & 65 & 68 & 50 & 50 & 50 & 63 & 69 \\
\hline Tesorería & 66 & 64 & 58 & 80 & 90 & 75 & 60 \\
\hline Movilidades & 14 & 13 & 12 & 15 & 13 & 14 & 13 \\
\hline Pago de honorarios & 15 & 12 & 15 & 13 & 13 & 11 & 13 \\
\hline
\end{tabular}

Fuente: elaboración propia. 
Con base en los resultados obtenidos se evidencia que son dos los procesos con calificación más baja o determinados como críticos (pago de honorarios y movilidades). Se propone lo siguiente:

1) Al revisar la trazabilidad de los procesos críticos se detecta que tienen demasiados pasos, por lo cual se sugiere revisarlos en coordinación con la Ugicu (Unidad de Gestión de la Calidad Universitaria) para que se responda en forma más adecuada y eficiente a las necesidades de los usuarios. Por lo tanto, la verdadera propuesta de valor es que estén articulados con el SGC.

2) Implementar una herramienta tecnológica (desarrollo interno) para seguimiento de tiempos y movimientos específicos tanto para los solicitantes como para los ejecutores funcionales.
Como producto de todo lo anterior se evidencia que los procesos del Área Financiera no están articulados con el SGC de la universidad, por lo cual se sugiere conformar un equipo de trabajo donde participen tanto los líderes del Área Financiera, quienes por su experticia deben proponer la optimización, como el personal de la Unidad de Calidad Universitaria, que es la encargada de mantener actualizados los procedimientos. Asíse podrá realizar la respectiva socialización, que ha de involucrar a todas las dependencias por ser procesos transversales a toda la institución.

La figura 1 muestra de nuevo todos los procesos considerados, incluidos los que presentan falencias significativas según el análisis de datos (movilidades y pago de honorarios), pero esta vez considerando los niveles establecidos en la tabla 1.

A partir de los resultados obtenidos se genera una propuesta de mejora descrita en la tabla 3.

Figura 1. Desempeño por proceso

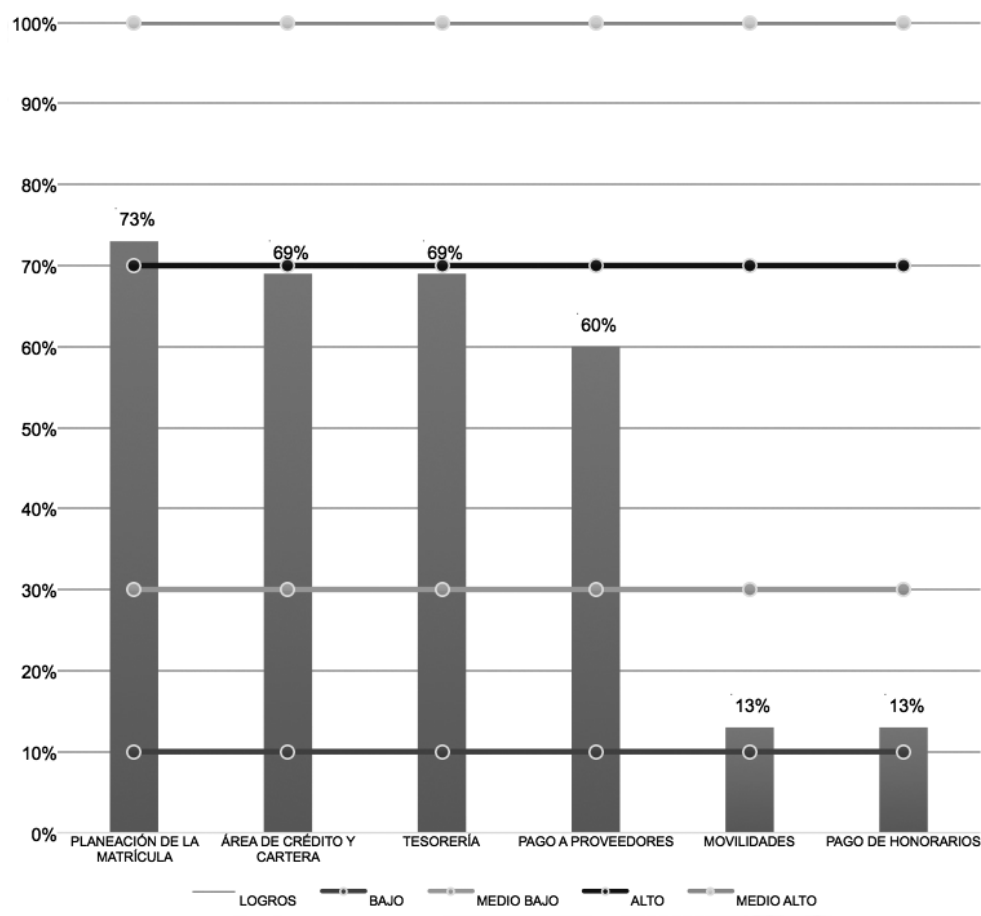

Fuente: elaboración propia. 
Tabla 3. Propuesta de mejora

\section{Recomendaciones para la mejora}

Con el fin de implementar un verdadero plan de mejora que permita evaluar los procesos del Área Financiera de la Usta y su articulación con el sistema de gestión de calidad, se propone en primer lugar documentar los procesos calificados como críticos (movilidades y pago de honorarios) según el desarrollo de esta investigación. Para esta actualización se requiere de un esquema metodológico que permita identificar cada una de las necesidades actuales de documentación o actualización de dichos procesos. En segundo lugar, en coordinación con la Ugicu han de revisarse los pasos involucrados (trazabilidad) para responda en forma más adecuada y eficiente a las necesidades de los diferentes usuarios, lo que permite la articulación de esos procesos con el SGC.

Después de socializar con la alta gerencia el resultado de la evaluación de los procesos, donde se evidenció el impacto tan alto en costos financieros, demostrado a través del modelo PEF tanto en términos económicos como en el deterioro de la imagen institucional, se espera implementar las siguientes acciones:
1) Programar jornadas de socialización y capacitación, tanto entre académicos como entre administrativos, para dar a conocer los procedimientos documentados. El objetivo es que sean interiorizados y permitan que el proceso sea óptimo y se pueda evidenciar la eficacia de las acciones planteadas en los ejercicios de auditoría tanto interna como externa.

2) Implementar una herramienta tecnológica, (desarrollo interno) que permita realizar el seguimiento a los tiempos y movimientos de los procesos, que deben ser específicos tanto para sus solicitantes como para sus ejecutores.

3) Generar estrategias de comunicación asertiva que incentiven el trabajo constructivo, colaborativo y eficiente entre funcionarios de diferentes dependencias que dan respuesta en conjunto a los mismos requerimientos

Actividades para ejecución del plan de mejora:

1) Se estableció que el perfil debe cumplir con una formación integral (actitud y conocimiento).

2) Se requiere de un rediseño radical (reingeniería) mediante el cual se logren mejoras sustanciales que permitan disminuir costos y aumentar la calidad del servicio y la rapidez en los procesos.

3) Se deben fortalecer la cohesión, el control y la articulación de las áreas involucradas en los procesos críticos (honorarios-movilidades).

4) Fuentes de verificación: hojas de ruta, planillas y sellos de recibido; también el seguimiento continuo servirá para realizar la trazabilidad de las solicitudes.

5. Responsables: directores de cada una de las dependencias administrativas mencionadas

Fuente: elaboración propia.

Asimismo, son relevantes las jornadas de capacitación a los involucrados en los procesos, que siempre conllevan resultados positivos, considerado el recurso humano como eje fundamental en el logro de las nuevas estrategias sugeridas a continuación:

- Implementar un software de seguimiento que logre intercomunicar las solicitudes de los académicos (de cualquier proceso) y la respuesta de las dependencias administrativas. Debe ser una herramienta de apoyo y seguimiento que evidencie o permita hacer la trazabilidad desde la fecha de solicitud hasta la de cierre o cumplimiento de lo solicitado.
- Realizar campañas impactantes de sensibilización acerca de la ruta y el manejo de la herramienta.

- Establecer los perfiles adecuados (tanto académicos como administrativos), que entiendan la importancia de la labor encomendada, la cual está encaminada a responder en forma oportuna y eficiente a los cambios a nivel tributario y normativo relacionados con las órdenes de prestación de servicios.

- Nombrar un equipo de mejora. El Área de Gestión Financiera es la encargada de realizar la socialización involucrando a todas las dependencias, por ser un proceso transversal a toda la universidad. 


\section{CONCLUSIONES}

La cultura organizacional de la IES estudiada constituye quizás el aspecto más representativo y visible de su particularidad y su identidad. Es así como el desconocimiento de las características de esa cultura dificulta en gran medida el logro de los objetivos propuestos como conclusión de este trabajo.

Aquíse logró proponer un plan de mejoramiento que permitirá identificar los beneficios económicos y financieros del sistema de gestión de la calidad y que garantizarála optimización en los procesos del Área Financiera de la IES.

Asimismo, mediante el análisis de los procesos del Área Financiera de la IES se logró identificar el costo de la calidad y la falta de calidad a través del modelo PEF.

En términos generales se evidenció la falta de articulación de los procesos del Área de Gestión Financiera con el sistema de gestión de calidad de la universidad. Se resalta que la importancia de este no está solo en implementarlo, sino en mantenerlo ya que con el tiempo los sistemas se desgastan. Por ende, se deben revisar permanentemente todos los frentes, entre ellos la articulación que debe mantener el SGC con todos los procesos de la institución.

Se evidenció también la falta de documentación de algunos procedimientos, que permitiría orientar las dinámicas del quehacer diario de cada proceso.

La presente investigación confirma que la principal barrera para implementar y mantener actualizado el sistema de gestión de la calidad es la resistencia al cambio. Así, el reto de los implementadores es la sensibilización previa como parte de la gestión del cambio, que permitirá afrontar los retos que se presenten, entre ellos el más relevante: la resistencia a aquel por parte de las personas involucradas en los procesos tanto administrativos como académicos.

Para contrarrestar tal resistencia se propone un par de lineamientos dentro de la universidad. Primero, generar estrategias de comunicación asertiva que incentiven el trabajo constructivo, colaborativo y eficiente entre funcionarios de diferentes dependencias que den respuesta en conjunto a los mismos requerimientos. Segundo, realizar la actualización documental de los procedimientos a través de un esquema metodológico, que permita identificar cada una de las necesidades actuales de documentación, no solo en función de las auditorias de calidad, sino también por tratarse de procedimientos fiscalizados tanto por auditorías internas como por la Revisoría Fiscal, además de ser procesos objeto de constante monitoreo, seguimiento y revisión.

Se propone impulsar un trabajo en equipo con base en planes encaminados a la articulación del SGC con los procesos objeto de este estudio para así lograr un beneficio tanto en los procesos funcionales de tipo administrativo como en la calidad del servicio prestado a la academia.

Los futuros investigadores podrían tener en cuenta los aspectos identificados en este trabajo en las diferentes IES para determinar la eficacia y eficiencia de estas en las áreas financieras.

Ahora bien, con base en la caracterización del proceso de gestión financiera, cuyo objetivo principal es evaluar y administrar de manera eficaz y eficiente la sostenibilidad financiera de la universidad, y para asegurar el desarrollo y bienestar de la comunidad universitaria, es prudente considerar y enfatizar que los procesos financieros deben hacer parte del sistema de gestión de la calidad articulándose en forma efectiva, lo cual aminorará las fallas en los mencionados procesos. 
La forma como se abordó la investigación puede ser un modelo de caso o una guía aplicable a otros procesos y también para otras universidades. En la Usta los procesos del Área Financiera se pueden evidenciar en los informes de las auditorías internas lideradas por la Ugicu.

\section{REFERENCIAS}

Águila, V. (2005). El concepto calidad en la educación universitaria: clave para el logro de la competitividad institucional. Revista Iberoamericana de Educación, 36(12), 1-7. Recuperado de https://rieoei.org/ RIE/article/view/2886

Gracia, S. y Dzul, L. A. (2007). Modelo PEF de costes de la calidad como herramienta de gestión en empresas constructoras: una visión actual. Revista Ingeniería de Construcción, 22(1), 43-56. Doi: https://dx.doi. org/10.4067/S0718-50732007000100005

Lozano, J. Keith, R. y Fonseca, I. (2014). Desarrollo e implementación de un sistema de costos de calidad en una empresa del sector automotriz que permite cuantificar y detectar las oportunidades de mejora. Industrial Data, 17 (1), 31-38.

Olmos, A. (2012). Modelos de calidad y gestión en educación superior, un análisis comparativo. Signos. Investigación en Sistemas de Gestión, 4(2), 83-96. Doi: https://doi.org/10.15332/s2145-1389.2012.0002.05

Pedraza, X., Rodriguez, Y. y Pérez, J. (2017). Medición de la gestión de la calidad universitaria: revisión bibliográfica. Signos. Investigación en Sistemas de Gestión, 9(1), 19-30. Doi: https://doi.org/10.15332/ s2145-1389.2017.0001.01

Perdomo, Á. (2010). Administración de los costos y gestión financiera de la calidad. Bogotá: Icontec.

Ruelas-Barajas, E. (1993). Calidad, productividad y costos. Salud Pública de México, 35(3), 298-304. Recuperado de http://saludpublica.mx/index.php/spm/ article/view/5660/6180

Satanova, A. y Sedliacikova, M. (2015). Model for controlling the total costs of quality. Procedia Economics and Finance, 26, 2-6. Doi: https://doi.org/10.1016/ S2212-5671(15)00792-3 